\title{
A INFLUÊNCIA DA INOVAÇÃO EM PRODUTOS E PROCESSOS NO DESEMPENHO DE EMPRESAS BRASILEIRAS
}

\author{
Natália Mendonça Terra \\ Mestre em Administração e Desenvolvimento Empresarial pela Universidade Estácio de Sá \\ nataliamterra@gmail.com (Brasil)
}

\section{Jose Geraldo Pereira Barbosa}

Doutorado em Administração pela Universidade Federal do Rio de Janeiro - UFRJ

Professor da Universidade Estácio de Sá

jose.geraldo@estacio.br (Brasil)

\section{Marco Aurélio Carino Bouzada}

Doutorado em Administração pelo Instituto Coppead de Administração - UFRJ

Professor titular da Universidade Estácio de Sá

marco.bouzada@estacio.br (Brasil)

\section{RESUMO}

O principal objetivo desse trabalho foi avaliar as relações entre desempenho de inovações em produtos (processos) e crescimento (lucratividade) de empresas brasileiras pertencentes a três setores: fabricação de máquinas e equipamentos; fabricação de produtos químicos e fabricação de equipamentos de informática, produtos eletrônicos e ópticos. Foram utilizados dados das pesquisas PINTEC (2003, 2005 e 2008) e PIA (2003 a 2010) coletados no Instituto Brasileiro de Geografia e Estatística - IBGE. Verificou-se, com base em resultados de regressões múltiplas e tratamento estatístico efetuado, a inexistência de uma relação positiva entre o desempenho de inovação em processo (produto) e lucratividade (crescimento). Em certa medida já se esperava que as inovações não fornecessem um nível de explicação alto para a variância em desempenho financeiro de empresas tendo em vista que a literatura indica outros fatores que também ajudam a explicar tal variância (atratividade do mercado, domínio de fontes de insumos, para citar apenas dois exemplos). Verificou-se também que a literatura visitada não é conclusiva sobre a relação entre inovação e desempenho financeiro, o que se deve em grande parte à diversidade de variáveis utilizadas na mensuração do desempenho tanto da inovação quanto financeiro. Além da rejeição das hipóteses da pesquisa, o trabalho revelou outros resultados relevantes que podem subsidiar o gestor no processo decisório relacionado ao processo de inovação.

Palavras-chave: Inovação em produto; Inovação em processo; Desempenho financeiro; Lucratividade; Crescimento. 


\section{INTRODUÇÃO}

Em tese, inovações em produtos possibilitam à empresa ampliar as vendas e aumentar sua receita pelo menos até o momento em que os competidores começam a responder procurando defender suas posições de mercado. Já as inovações em processos podem reduzir os custos dos métodos de produção em relação aos de seus concorrentes, o que pode significar um aumento na lucratividade da empresa. Entretanto, os resultados de pesquisas sobre a relação entre inovação e desempenho financeiro de empresas continuam gerando resultados controversos. Grande parte dos estudos empíricos apoia apenas parcialmente (Brito, Brito \& Morganti, 2009; Miranda, Gallon \& Nogueira, 2011; Mauro \& Brito, 2011; Kemp, Folkeringa, Jong \& Wubben, 2003; Boscolo \& Sbragia, 2008) a existência de uma relação positiva entre inovação e desempenho empresarial, não conseguindo confirmar a relação a parir de todas as variáveis testadas.

Assim, o objetivo principal desse trabalho foi avaliar a influência do desempenho de inovações em produtos e processos no desempenho financeiro de empresas brasileiras. Para isso foi necessário, como objetivos intermediários, identificar os desempenhos em inovação em produtos e em processos e os indicadores de crescimento e lucratividade das empresas pesquisadas. Como objetivos secundários do trabalho, procurou-se comparar a influência do desempenho de inovações em processo (produto) na lucratividade (crescimento) das empresas (i) entre os anos 2003, 2005 e 2008 e (ii) entre três setores da economia brasileira. As hipóteses testadas foram: 1. Mantendo outros aspectos constantes, quanto maior o desempenho de inovações em processos, maior será a lucratividade da empresa e 2. Mantendo outros aspectos constantes, quanto maior o desempenho de inovações em produtos, maior será o crescimento da empresa. É importante ressaltar a relativização das hipóteses, já que, por exemplo, pode ser que haja um subsegmento com maior lucratividade potencial e que, ao mesmo tempo, seja mais propenso a empresas mais inovadoras em processos. Mas, para fins de fluidez de texto, nas futuras menções às hipóteses de pesquisa, este trecho que as relativiza ("Mantendo os outros aspectos constantes") foi suprimido. 


\section{REVISÃO DE LITERATURA}

\subsection{Inovação}

Para a Organização para Cooperação e Desenvolvimento Econômico (OCDE) (2005, p.57), "uma inovação de produto é a introdução de um bem ou serviço novo ou significativamente melhorado no que concerne às suas características ou usos previstos." enquanto que "uma inovação de processo é a implementação de um método de produção ou distribuição novo ou significativamente melhorado" (OCDE, 2005, p.58).

Entretanto, frequentemente um entendimento errôneo do processo de inovação impede que se perceba a intensa relação que existe entre inovação em produto/serviço e a inovação em processo para sua produção/prestação. Ou seja, inovações em produtos frequentemente levam a inovações em seus processos de produção e vice-versa (Tidd. Bessant \& Pavitt, 2005).

Usualmente as inovações são diferenciadas por seu grau de mudança em relação aos produtos e processos que existiam anteriormente, sendo classificadas em: i) inovações incrementais, que possuem baixo grau de novidade em relação à versão anterior do produto ou processo; e ii) inovações radicais que possuem alto grau de novidade, pois rompem as trajetórias existentes, inaugurando uma nova rota tecnológica (Tigre, 2006).

Arbix (2010) menciona que invenção e inovação podem estar altamente relacionadas, porém constituem fenômenos distintos, capazes de gerar diferentes dinâmicas econômicas. É certo que uma inovação pode nascer de uma invenção. Mas, diferentemente da invenção, a inovação precisa encontrar seu desenlace em um produto, processo ou serviço no mercado. Portanto, na economia real a inovação se refere a uma primeira comercialização de uma ideia ou projeto; por isso mesmo, seu locus privilegiado é a empresa, capaz de interligar desenvolvimento, produção e a comercialização.

\subsection{Desempenho de inovações}

“A avaliação do desempenho da inovação é um dos aspectos de gestão mais importante e desafiador para o sucesso das organizações atuais, e tal tarefa ainda torna-se mais complexa quando relacionadas às características inovadoras das organizações." (Oliveira, Clemente \& Caulliraux, 2009, p.1). O Manual de Oslo destaca a dificuldade de mensurar um processo dinâmico como a inovação. As empresas realizam continuamente mudanças em produtos e processos, buscando novos conhecimentos para se chegar a uma inovação. Esta mensuração fica ainda mais complicada em empresas que 
desenvolvem atividades de inovação caracterizadas por mudanças pequenas e incrementais em oposição às inovações radicais.

Para Tidd e Bessant (2009), diversos fatores influenciam a capacidade de uma empresa de se beneficiar comercialmente de suas inovações: capacidade de manter segredo industrial; inovação decorrente de conhecimento tácito acumulado; lead times (alta velocidade de entrada no mercado) e serviço pós-venda; curva de aprendizado na produção; posse de ativos complementares (marketing e produção); complexidade da inovação; criação de padrões; e capacidade de apropriação (propriedade intelectual). De acordo com a OCDE (2005), o sucesso de uma inovação depende de uma série de fatores, sendo um deles a qualidade percebida desta inovação. Igualmente, o resultado das inovações em processo pode depender de mudanças organizacionais para tirar vantagem desses novos processos.

Pode-se também argumentar que, em longo prazo, o desempenho de determinada inovação é verificada em termos do crescimento de sua penetração no mercado ao longo dos anos que se sucederam à sua introdução. Essa medida de adoção, de formato em $\mathrm{S}$ (curva de epidemias), assim como sua direção (perfil do grupo adotante), é influenciada por vários fatores: (i) vantagem relativa: é o grau em que uma inovação é percebida como melhor que o produto que ela substitui ou concorre. Fatores financeiros e não financeiros ditam tal vantagem; (ii) compatibilidade: é o grau em que uma inovação é percebida como sendo consistente com os valores, experiência e necessidades presentes dos adotantes potenciais; (iii) complexidade: é o grau em que uma inovação é percebida como sendo difícil de entender ou usar; (iv) possibilidade de ser experimentada: uma inovação que pode ser experimentada representa menores incertezas para os adotantes potenciais e permite o "aprender fazendo"; e (v) visibilidade: é o grau com que os resultados de uma inovação são visíveis a outros (Rogers, 2003).

Tidd, Bessant e Pavitt (2005) argumentam que a trajetória de inovação tecnológica de uma organização depende do setor econômico em que o negócio se situa, e inspirando-se na tipologia de Freeman e Soete (1997), classificaram os setores em: (i) setores dominados por fornecedores (comandados por fornecedores); (ii) setores intensivos em escala; (iii) setores baseados em ciência, como é o caso do setor de fabricação de produtos químicos, objeto de estudo da presente pesquisa; (iv) setores intensivos em informação; e (v) setores fornecedores especializados (comandados por clientes), como é o caso do setor de fabricação de máquinas e equipamentos e do setor de fabricação de equipamentos de informática, produtos eletrônicos e ópticos, também objetos de estudo da presente pesquisa.

Embora concorde com as proposições de Freeman e Soete (1997), Malerba (2002) sugere maiores pesquisas sobre o relacionamento entre instituições nacionais (contexto institucional) e 
sistemas setoriais de inovação e produção. Afirma ele que o sistema de patentes, os direitos de propriedade ou as leis antimonopólios produzem diferentes efeitos sobre diferentes setores da indústria. Frequentemente as características de instituições nacionais favorecem determinados setores industriais que se enquadram melhor às especificidades das instituições. Não é de se admirar, portanto que, em certos países, um determinado setor industrial torna-se predominante exatamente porque as instituições nacionais daquele país produziram um ambiente mais adequado para o mesmo.

\subsection{Indicadores de desempenho financeiro}

Nesta seção procurou-se identificar os indicadores financeiros mais utilizados para calcular a lucratividade das empresas sem a preocupação de relacioná-los com a inovação. Tal relação é discutida na seção seguinte.

Brigham e Houston (1999) mencionam que o valor econômico agregado (EVA) seria uma forma de medir a verdadeira rentabilidade de uma operação. Anthony e Govindarajan (2008) reforçam os benefícios de se utilizar o percentual do retorno sobre o investimento (ROI) e o EVA para avaliar diferentes unidades de negócio. Essas duas medidas relacionam o lucro com a base de investimentos. Os autores explicam que o EVA é conceitualmente superior ao ROI. Porém, fica claro em suas pesquisas que o ROI é mais utilizado nos negócios que o EVA.

Moretti e Sanchez (2011) em pesquisa que teve por objetivo verificar o desempenho das empresas do Grande ABC entre os anos de 2001 a 2008 usaram três medidas de desempenho: o retorno sobre o ativo (ROA); retorno operacional sobre ativos (OROA); e o retorno sobre o patrimônio líquido (ROE). Na área da inovação, Miranda et al. (2011) analisaram a influência dos investimentos em ativos intangíveis e do grau de inovação no desempenho econômico das empresas participantes dos setores mais inovadores do Brasil, segundo a classificação do Índice Brasil de Inovação (IBI). Para isto, a pesquisa utilizou os seguintes indicadores de desempenho econômico: ROE, ROA, retorno sobre vendas (ROS), rentabilidade do patrimônio líquido (RPL) e grau de intangibilidade (GI).

Andia, Garcia e Bacha (2012), Macedo, Sousa, Souza e Cípola (2009), Veloso e Malik (2010), Krauter (2004) e inúmeras outras pesquisas que tiveram por objetivo a verificação da eficácia econômica empresarial nos mais diversos ramos de atividades utilizaram, principalmente, os indicadores ROE, ROI, ROA e RPL para verificar este desempenho. De acordo com Rezende, Duarte, Silva e Silva (2010 como citado em Matarazzo, 2010), os índices que representam melhor a situação econômica de uma empresa são os índices de rentabilidade.

Revista de Administração e Inovação, São Paulo, v. 12, n.3 p. 183-208, jul./set. 2015. 
De acordo com os parágrafos acima, o ROE e ROA foram os indicadores mais utilizados para aferição de lucratividade, sendo o último escolhido para efeito desta pesquisa, tendo em vista as informações disponibilizadas pelo IBGE para este estudo.

\subsection{Relação entre desempenho financeiro e inovação}

Campos e Ruiz (2009) argumentam que os estudos dos padrões setoriais de inovação se justificam porque possibilitam identificar diferenças setoriais em termos de nível de investimentos em inovação, resultados alcançados e o impacto das inovações no mercado. Assim, para Tidd, Bessant e Pavitt (2005), com relação ao posicionamento estratégico em termos de inovação, o setor de fabricação de produtos químicos procura desenvolver produtos tecnologicamente relacionados, por meio do aproveitamento de ciência básica (biologia molecular) e obtenção de ativos complementares (marketing, produção). Por sua vez, os setores de fabricação de máquinas e equipamentos e de fabricação de equipamentos de informática, produtos eletrônicos e ópticos procuram monitorar e responder às necessidades de usuários, adaptando mudanças tecnológicas às necessidades dos usuários e mantendo fortes relações com usuários pioneiros.

Por outro lado, Mintzberg, Ahlstrand e Lampel (2005) afirmam que a ação gerencial específica de cada empresa seria o fator determinante na explicação da lucratividade, embora também aceitem que uma proporção apreciável da variância da lucratividade de empresas possa ser explicada por características setoriais, um dos principais argumentos a sustentar a proposição por Porter (2005) do conceito de grupos estratégicos.

No estudo de Miranda et al. (2011), o objetivo foi identificar a existência de relação entre a inovação e o desempenho econômico empresarial (medido por meio dos indicadores ROE, ROA, ROS, RPL e GI) de empresas listadas na BM\&FBovespa e participantes do ranking setorial de inovação proposto pelo IBI. Os autores utilizaram ativos intangíveis (investimentos em marketing não rotineiro, treinamento, software e despesas correntes em P\&D entre outros) como proxy da inovação. Foi constatada uma relação positiva entre investimentos em ativos intangíveis e alguns indicadores econômicos, mas nenhuma evidência pode ser concluída sobre a contribuição do grau de inovação para a melhoria no desempenho econômico empresarial.

Brito et al. (2009) publicaram um estudo semelhante, com foco no setor químico, utilizando dados do construto inovação da Pesquisa Industrial de Inovação Tecnológica - PINTEC e dados do desempenho financeiro das empresas coletados do Balanço anual da Gazeta Mercantil, para os anos de 1999 e 2001. Foram desenvolvidas duas hipóteses de pesquisa do estudo: H1: Quanto maior o 
investimento em inovação, maior a taxa de crescimento da empresa e H2: Quanto maior o investimento em inovação, maior a lucratividade da empresa. Para mensurar o investimento em inovação foram utilizados os seguintes indicadores: gastos em P\&D, patentes, inovações significativas, pesquisas de inovação, anúncio de produtos, empregados devotados à inovação, etc. Os resultados mostraram que as variáveis de inovação não explicaram a variabilidade dos indicadores de lucratividade das empresas, mas explicaram parte relevante da variabilidade da taxa de crescimento da receita líquida, corroborando com os achados do modelo 3 de Miranda et al. (2011) que também trataram de investimentos em inovação.

Em um sentido mais amplo, Mauro e Brito (2011) investigaram o efeito das práticas operacionais de 47 empresas do setor sucroenergético do estado de São Paulo no seu desempenho financeiro percebido. Entre essas práticas estão incluídas as boas práticas em inovação. Entre outros fatores, a utilização de boas práticas em inovação apresentou relação com o crescimento do faturamento, mas não com a lucratividade, de forma similar aos resultados encontrados na pesquisa de Brito et al. (2009).

Por sua vez, no seu estudo, Queiroz (2010) analisou o impacto do crescimento dos gastos em P\&D na taxa de crescimento dos lucros das empresas de acordo com o modelo OJ (estabelecido a partir das iniciais dos autores Ohlson e Juettner e que tem como princípio central que o crescimento nos lucros explica o índice preço/lucros esperados). O autor informa que os investimentos em P\&D podem ser classificados como "ativos de inovação". Os resultados de seu estudo não apresentaram evidências para o mercado brasileiro da existência de uma relação, positiva e estatisticamente significante, entre os gastos com P\&D e o crescimento dos lucros das empresas em curto prazo no Brasil. Uma das explicações para o resultado obtido pode ser o tratamento contábil/tributário que era dispensado a esse tipo de gasto na legislação brasileira, que admitia uma abordagem dual nos gastos de P\&D (ativo ou despesa).

Kemp et al. (2003) testaram a relação entre a inovação e o desempenho da empresa, levando em conta o tamanho das empresas estudadas. A avaliação de desempenho baseou-se em quatro variáveis: crescimento dos lucros, crescimento do emprego, crescimento das vendas e crescimento da produtividade. A inovação foi verificada através de 24 variáveis, sendo que algumas são similares àquelas utilizadas por Brito et al. (2009): \% de empregados devotados à inovação, \% de cooperação com outras empresas, \% de patentes, \% de tempo gasto com inovação pelos contratados etc. Os autores verificaram que as únicas variáveis com efeitos positivos foram o crescimento do emprego e o crescimento das vendas, sendo que os lucros e a produtividade não são influenciados significativamente pelo output da inovação.

Revista de Administração e Inovação, São Paulo, v. 12, n.3 p. 183-208, jul./set. 2015. 
Malachias e Meirelles (2009), a partir de resultados de um estudo aplicado a empresas de tecnologia da informação, sugerem que a inovação ocasionou aumentos de market share, patrimônio líquido per capita e ativo fixo per capita. O desempenho inovativo foi avaliado pelos seguintes indicadores: aumento do mix de serviços ou produtos, aumento na participação do mercado, redução do custo de trabalho e vendas de serviços novos ou aperfeiçoados.

Da mesma forma Camara e Galão (2006) analisaram o impacto da inovação e da orientação para o mercado sobre o desempenho das empresas do arranjo produtivo local de Londrina. Para os autores, o impacto da inovação se devia à implementação de inovações de produto e processo, inovações organizacionais e atividades inovativas, variáveis essas que foram identificadas nas empresas pesquisadas. A análise de correlação realizada permitiu verificar que, de forma geral, a inovação e a orientação para o mercado conduzem a um desempenho superior e estão contribuindo para o crescimento do faturamento e para o retorno do investimento nas indústrias pesquisadas.

Num estudo qualitativo realizado por Benedetti (2006) e aplicado em pequenas empresas do setor de panificação, verificou-se como essas empresas de fato utilizam a inovação como forma de crescimento dos negócios. Os proprietários entrevistados constataram que ações voltadas à inovação, como mudanças estruturais, diferenciação e diversificação do mix de produtos e serviços, requalificação de funcionários e novo foco no atendimento às necessidades dos clientes, contribuíram de forma relevante para o crescimento de suas empresas. Os resultados da pesquisa de Boscolo e Sbragia (2008) ofereceram apoio parcial à hipótese de que o desempenho médio de empresas inovadoras, medido por meio de indicadores de rentabilidade, criação de valor e de domínio de mercado, é superior ao desempenho médio de empresas convencionais.

A teoria visitada mostrou que são utilizados diferentes tipos de indicadores para se medir a inovação, de acordo com os objetivos específicos de cada pesquisa e as informações possíveis de serem coletadas.

\section{METODOLOGIA}

Quanto aos fins a pesquisa é explicativa. Segundo Vergara (2009) a investigação explicativa visa esclarecer quais fatores contribuem de alguma forma, para a ocorrência de determinado fenômeno. Esse foi o caso da presente pesquisa que teve como principal objetivo avaliar as relações entre desempenho de inovações em produtos (processos) e crescimento (lucratividade) de empresas 
brasileiras pertencentes a três setores: fabricação de máquinas e equipamentos; fabricação de produtos químicos e fabricação de equipamentos de informática, produtos eletrônicos e ópticos.

Quanto aos meios, a pesquisa foi classificada como documental, pois os dados foram retirados de documentos oficiais do Instituto Brasileiro de Geografia e Estatística - IBGE. A abordagem da pesquisa foi de natureza quantitativa. A população neste estudo foi definida por todas as empresas brasileiras pertencentes aos três setores da indústria de transformação que tiveram maior quantidade de empresas respondentes na PINTEC 2008, dentre os setores mais inovadores segundo a mesma pesquisa. São eles: 1. Fabricação de máquinas e equipamentos; 2. Fabricação de produtos químicos e 3. Fabricação de equipamentos de informática, produtos eletrônicos e ópticos.

Após a união das bases da Pesquisa Industrial Anual - PIA e PINTEC, eliminação de faltantes e observações aberrantes, a amostra para a análise incluiu 81 empresas no total do Setor 1 - Fabricação de máquinas e equipamentos, 76 empresas no total do Setor 2 - Fabricação de produtos químicos e 60 empresas no total do Setor 3 - Fabricação de equipamentos de informática, produtos eletrônicos e ópticos, totalizando 217 empresas.

Para obter acesso aos dados secundários das pesquisas PINTEC e PIA, foi necessária a submissão de um projeto de pesquisa para análise técnica do IBGE. Após aprovação do projeto, a coleta dos dados foi realizada em cinco visitas a uma "sala de sigilo" nas dependências do próprio IBGE.

A coleta de dados sobre o desempenho de inovação em produtos e processos foi realizada através dos dados disponíveis nas PINTECs dos anos de 2003, 2005 e 2008, para os três setores definidos. A Tabela 1 apresenta os indicadores que foram coletados para todas as empresas da amostra e seus respectivos códigos.

\section{Tabela 1}

Variáveis de desempenho de inovações em produtos e processos implementadas entre o ano (A - 2) e o ano A (ano de cada PINTEC)

\begin{tabular}{|c|l|c|}
\hline $\begin{array}{c}\text { Tipo de } \\
\text { Inovação }\end{array}$ & \multicolumn{1}{|c|}{ Variáveis explicativas - Descrição } & $\begin{array}{c}\text { Código } \\
\text { PINTEC }\end{array}$ \\
\hline \multirow{5}{*}{ Produto } & $\begin{array}{l}\text { Participação de produto novo ou significativamente aprimorado para a } \\
\text { empresa, mas já existente no mercado nacional, nas vendas líquidas internas }\end{array}$ & V85 \\
\cline { 2 - 3 } & $\begin{array}{l}\text { Participação de produto novo ou significativamente aprimorado para a } \\
\text { empresa, mas já existente no mercado nacional, nas exportações }\end{array}$ & V89 \\
\cline { 2 - 4 } & $\begin{array}{l}\text { Participação de produto novo ou significativamente aprimorado para o } \\
\text { mercado nacional, mas já existente no mercado mundial, nas vendas líquidas } \\
\text { internas }\end{array}$ & V86 \\
\cline { 2 - 4 } & $\begin{array}{l}\text { Participação de produto novo ou significativamente aprimorado para o } \\
\text { mercado nacional, mas já existente no mercado mundial, nas exportações }\end{array}$ & V90 \\
\cline { 2 - 4 } & $\begin{array}{l}\text { Participação de produto novo para o mercado mundial nas vendas líquidas } \\
\text { Pand }\end{array}$ & V87 \\
\hline
\end{tabular}

Revista de Administração e Inovação, São Paulo, v. 12, n.3 p. 183-208, jul./set. 2015. 
A influência da inovação em produtos e processos no desempenho de empresas brasileiras

\begin{tabular}{|c|c|c|}
\hline & internas & \\
\hline & Participação de produto novo para o mercado mundial nas exportações & V91 \\
\hline & $\begin{array}{l}\text { Importância das inovações em produto para a melhora da qualidade dos bens } \\
\text { ou serviços }\end{array}$ & V93 \\
\hline & $\begin{array}{l}\text { Importância das inovações em produto para a ampliação da gama de bens ou } \\
\text { serviços ofertados }\end{array}$ & V94 \\
\hline \multirow{7}{*}{ Processo } & $\begin{array}{l}\text { Importância das inovações em processo para o aumento da capacidade de } \\
\text { produção ou de prestação de serviços }\end{array}$ & V98 \\
\hline & $\begin{array}{l}\text { Importância das inovações em processo para o aumento da flexibilidade da } \\
\text { produção ou da prestação de serviços }\end{array}$ & V99 \\
\hline & $\begin{array}{l}\text { Importância das inovações em processo para a redução dos custos de } \\
\text { produção ou dos serviços prestados }\end{array}$ & V100 \\
\hline & $\begin{array}{l}\text { Importância das inovações em processo para o redução dos custos do } \\
\text { trabalho }\end{array}$ & V101 \\
\hline & $\begin{array}{l}\text { Importância das inovações em processo para a redução do consumo de } \\
\text { matérias-primas }\end{array}$ & V102 \\
\hline & $\begin{array}{l}\text { Importância das inovações em processo para a redução do consumo de } \\
\text { energia }\end{array}$ & V103 \\
\hline & Importância das inovações sem processo para a redução do consumo de água & V104 \\
\hline
\end{tabular}

Os seis primeiros indicadores de inovações em produtos são apresentados na PINTEC por meio da participação das respectivas inovações no total de vendas líquidas internas e exportações. Os demais indicadores de produtos e processos são apresentados na PINTEC através da medição do seu grau de importância, indicado por alto, médio, baixo e não relevante. A rigor a PINTEC entende que esses indicadores medem os impactos das inovações em produtos e processos. O entendimento dos autores do presente estudo de que esses impactos em certa medida refletem o desempenho da empresa em termos de crescimento e lucratividade constitui, portanto uma limitação do método.

Já a coleta de dados sobre o desempenho financeiro das empresas foi realizada através dos dados disponíveis nas PIAs dos anos de 2003 a 2010, referente aos setores definidos. Na Tabela 2, estão descritos os indicadores de desempenho financeiro retirados da base de dados da PIA, com seus respectivos códigos, e que foram utilizados para o cálculo das variáveis dependentes.

Tabela 2

Variáveis dependentes, indicadores e respectivos códigos

\begin{tabular}{|c|l|c|}
\hline $\begin{array}{c}\text { Variável } \\
\text { dependente }\end{array}$ & Indicadores - Descrição & $\begin{array}{c}\text { Código } \\
\text { PIA }\end{array}$ \\
\hline \multirow{2}{*}{\begin{tabular}{l} 
Lucratividade \\
\cline { 2 - 3 }
\end{tabular}} & \begin{tabular}{l} 
Lucro do Resultado do exercício antes da distribuição de \\
imposticipações, da contribuição social e da provisão para o \\
\cline { 2 - 3 }
\end{tabular} & V0074 \\
\hline Crescimento & Receita Líquida de Vendas & V0210 \\
\hline
\end{tabular}

Revista de Administração e Inovação, São Paulo, v. 12, n.3 p. 183-208, jul./set. 2015. 


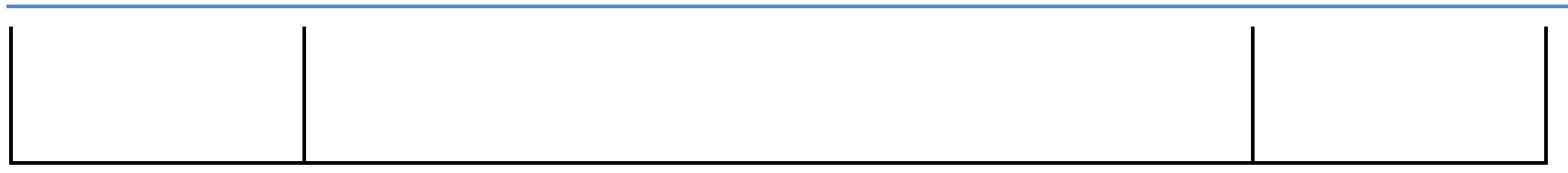

De posse do Lucro do Resultado do Exercício e do Total do ativo das empresas dos setores pesquisados referente aos anos abrangidos na pesquisa, foi calculado o ROA (Lucro do Resultado do Exercício/ Total do ativo) de todas as empresas, sendo este o indicativo de lucratividade das empresas utilizado na pesquisa.

De posse da Receita Líquida de Vendas das empresas dos setores pesquisados referente aos anos abrangidos na pesquisa, foi calculada a taxa de crescimento (Receita Líquida de Vendas Atual Receita Líquida de Vendas do ano anterior/ Receita Líquida de Vendas do ano anterior) de todas as empresas, sendo este o indicativo de crescimento das empresas utilizado na pesquisa.

A Seguir, foram realizadas diversas regressões lineares (17 para cada setor), tentando explicar a lucratividade e o crescimento das empresas em diferentes anos em função, respectivamente, dos indicadores de desempenho de inovação em processo e produto.

A Tabela 3 sumariza as 51 regressões múltiplas que foram realizadas, com a seguinte representação: (variável explicativa) x (variável dependente), de acordo com a legenda a seguir:

$P_{C}=$ Indicadores de desempenho de inovações em processo implementadas entre o ano (A 2) e o ano A (da PINTEC)

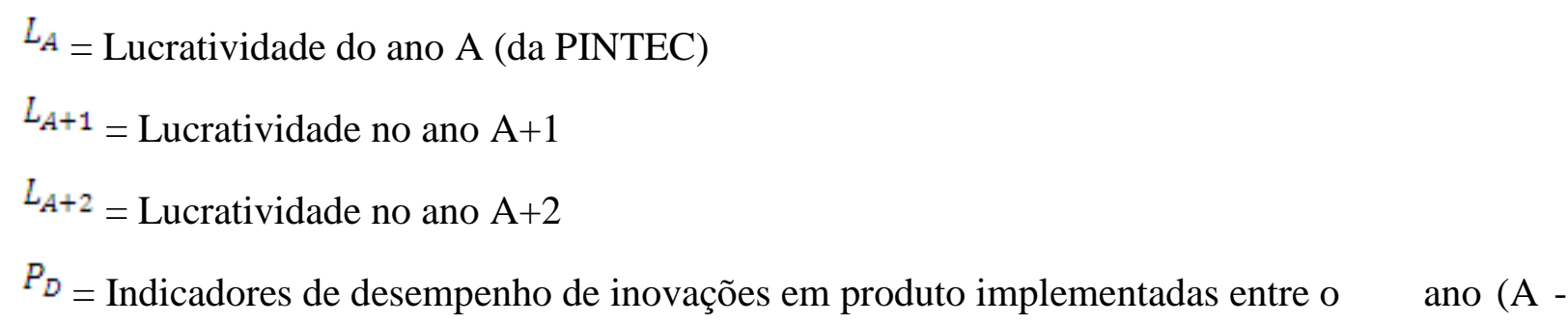
2) e o ano A (da PINTEC)

$$
\begin{aligned}
& C_{A}=\text { Crescimento no ano A (da PINTEC) } \\
& C_{A+1}=\text { Crescimento no ano } \mathrm{A}+1 \\
& C_{A+2}=\text { Crescimento no ano } \mathrm{A}+2
\end{aligned}
$$

\section{Tabela 3}

Sumário das regressões múltiplas realizadas

\begin{tabular}{|l|l|l|l|}
\hline SETORES & 2003 & 2005 & 2008 \\
\hline \multirow{3}{*}{ S1 } & $P_{C} X L_{A} ;$ & $P_{C} X L_{A} ; P_{C} X L_{A+1} ; P_{C} X L_{A+2} ;$ & $P_{C} X L_{A} ; P_{C} X L_{A+1} ; P_{C} X L_{A+2} ;$ \\
& $P_{C} X L_{A+1} ; P_{C} X L_{A+2} ;$ & $P_{D} X C_{A} ; P_{D} X C_{A+1} ; P_{D} X C_{A+2}$ & $P_{D} X C_{A} ; P_{D} X C_{A+1} ; P_{D} X C_{A+2}$ \\
\hline
\end{tabular}


A influência da inovação em produtos e processos no desempenho de empresas brasileiras

\begin{tabular}{|c|c|c|c|}
\hline S2 & $\begin{array}{l}P_{C} X L_{A} ; \\
P_{C} X L_{A+1} ; P_{C} X L_{A+2} \\
P_{D} X C_{A+1} ; P_{D} X C_{A+2}\end{array}$ & $\begin{array}{l}P_{C} \mathrm{XI}_{A} ; P_{C} \mathrm{XL}_{A+1} ; P_{C} \mathrm{XL}_{A+2} ; \\
P_{D} \mathrm{X} C_{A} ; P_{D} \mathrm{X} C_{A+1} ; P_{D} \mathrm{X} C_{A+2}\end{array}$ & $\begin{array}{l}P_{C} X L_{A} ; P_{C} X L_{A+1} ; P_{C} X L_{A+2} ; \\
P_{D} X C_{A} ; P_{D} X C_{A+1} ; P_{D} X C_{A+2}\end{array}$ \\
\hline S3 & $\begin{array}{l}P_{C} X_{L_{A}} ; \\
P_{C} X L_{A+1} ; P_{C} X L_{A+2} ; \\
P_{D} X C_{A+1} ; P_{D} X C_{A+2}\end{array}$ & $\begin{array}{l}P_{C} X \mathbb{L}_{A} ; P_{C} X L_{A+1} ; P_{C} X L_{A+2} ; \\
P_{D} X C_{A} ; P_{D} X C_{A+1} ; P_{D} X C_{A+2}\end{array}$ & $\begin{array}{l}P_{C} X L_{A} ; P_{C} X \mathbb{L}_{A+1} ; P_{C} X L_{A+2} ; \\
P_{D} X C_{A} ; P_{D} X C_{A+1} ; P_{D} X C_{A+2}\end{array}$ \\
\hline
\end{tabular}

A análise, para atender ao objetivo principal da pesquisa, foi efetuada a partir de considerações sobre os 51 coeficientes de determinação R-Quadrado e sobre as variáveis explicativas com valores-P relevantes $(<10 \%)$.

Outra importante limitação da metodologia é que os desempenhos de empresas (crescimento e lucratividade) em cada ano foram relacionados apenas aos desempenhos de suas inovações implementadas entre o ano em que ocorreu a PINTEC justamente anterior e dois anos antes da PINTEC em questão.

\section{ANÁLISE E DISCUSSÃO DOS RESULTADOS}

\subsection{Tratamento dos dados e análise dos resultados}

\subsubsection{Resultados das regressões múltiplas.}

Visando testar a primeira hipótese, foram realizadas 9 regressões para cada setor estudado, utilizando como variáveis explicativas os indicadores de desempenhos de inovações em processos dos anos de 2003, 2005 e 2008 e como variável dependente a lucratividades das empresas, medida pelo retorno sobre o ativo - ROA de 2003 a 2010.

Objetivando testar a segunda hipótese, foram realizadas 8 regressões para cada setor estudado, utilizando como variáveis explicativas os indicadores de desempenhos de inovações em produtos dos anos de 2003, 2005 e 2008 e como variável dependente a taxa de crescimento das empresas de 2004 a 2010.

A título de ilustração, são mostrados na Tabela 4 os resultados mais satisfatórios (maior nível de explicação) para o Setor 1- Fabricação de máquinas e equipamentos, dentre as oito regressões 
referentes a este setor, relacionando os indicadores de desempenho de inovação em produto e a taxa de crescimento. Ressalte-se que a regressão apresentada é apenas 1 das 51 realizadas.

\section{Tabela 4}

Setor 1: Indicadores de desempenho de inovação em produto de $2003 \mathrm{X}$ taxa de crescimento no ano A+2

\begin{tabular}{|c|c|c|c|c|}
\hline \multicolumn{2}{|c|}{ Estatística de regressão } & & & \\
\hline R múltiplo & 0,32 & & & \\
\hline R-Quadrado & 0,10 & & & \\
\hline \multicolumn{5}{|l|}{ R-quadrado } \\
\hline Erro padrão & 0,32 & & & \\
\hline Observações & 81 & & & \\
\hline \multicolumn{5}{|l|}{ ANOVA } \\
\hline & Gl & $\mathbf{F}$ & $\begin{array}{c}\text { F de } \\
\text { significação }\end{array}$ & \\
\hline Regressão & 8 & 1,03 & 0,42 & \\
\hline Resíduo & 72 & & & \\
\hline \multirow[t]{2}{*}{ Total } & 80 & & & \\
\hline & Coeficientes & valor-P & $\begin{array}{c}95 \% \\
\text { inferiores }\end{array}$ & $\begin{array}{c}95 \% \\
\text { superiores }\end{array}$ \\
\hline Interseção & $9,77 \%$ & 0,45 & $-0,16$ & 0,35 \\
\hline V85 & $-0,13 \%$ & 0,65 & $-0,01$ & 0,00 \\
\hline V89 & $0,23 \%$ & 0,42 & 0,00 & 0,01 \\
\hline V86 & $-0,27 \%$ & 0,47 & $-0,01$ & 0,00 \\
\hline V90 & $0,13 \%$ & 0,67 & 0,00 & 0,01 \\
\hline V87 & $0,84 \%$ & 0,02 & 0,00 & 0,02 \\
\hline V91 & $-15,14 \%$ & 0,36 & $-0,48$ & 0,18 \\
\hline V93 & $-2,35 \%$ & 0,49 & $-0,09$ & 0,04 \\
\hline V94 & $-0,36 \%$ & 0,91 & $-0,07$ & 0,06 \\
\hline
\end{tabular}

Os resultados indicam que pelo R-Quadrado não é possível explicar satisfatoriamente a relação no ano de 2003 entre as variáveis de desempenho de inovação em produto e o crescimento no ano de $2005(\mathrm{~A}+2)$. Para a análise estatística foi definido nesta pesquisa um nível de significância de $10 \%$. Portanto, com relação ao valor-P, foi encontrada uma variável relevante, a V87 (Participação de produto novo para o mercado mundial, nas vendas líquidas internas). Para cada aumento unitário na V87, a taxa de crescimento é aumentada em 0,84 pontos percentuais.

\subsubsection{Análise dos coeficientes de determinação (R-Quadrados) e dos valores-P}

Os R-Quadrados evidenciam a força da relação entre as variáveis de desempenho de inovação em processo (produto) nos anos de cada pesquisa PINTEC, e a lucratividade (crescimento) no ano de cada pesquisa e nos dois que se sucederam. Para um melhor entendimento, são apresentados nas 
A influência da inovação em produtos e processos no desempenho de empresas brasileiras

Tabelas 5, 6 e 7 um resumo de todos os R-Quadrados encontrados nas 17 regressões realizadas em cada um dos três setores estudados. Entretanto, serão comentados em função do espaço disponível, e a título de ilustração, apenas os R-Quadrados do Setor 1 - Fabricação de máquinas e equipamentos.

Já em relação aos valores-P, não é viável apresentar todos eles, já que cada variável, em cada uma das 51 regressões, apresenta seu próprio valor-P. No entanto, serão comentados nas seções a seguir, uma para cada um dos 3 setores, as influências das variáveis que apresentaram valor $\mathrm{P}<10 \%$.

\section{Setor 1 - Fabricação de máquinas e equipamentos}

\section{Tabela 5}

R-Quadrados do Setor 1 - Fabricação de máquinas e equipamentos

\begin{tabular}{|c|c|c|c|}
\hline REGRESSÕES & $\mathbf{2 0 0 3}$ & $\mathbf{2 0 0 5}$ & $\mathbf{2 0 0 8}$ \\
\hline$P_{C} \mathrm{X} L_{A}$ & 0,06 & 0,11 & 0,06 \\
\hline$P_{C} \mathrm{X} L_{A+1}$ & 0,05 & 0,08 & 0,07 \\
\hline$P_{C} \mathrm{X} L_{A+2}$ & 0,02 & 0,08 & 0,08 \\
\hline$P_{D} \mathrm{X} C_{A}$ & $\begin{array}{c}\text { Regressão não } \\
\text { realizada }\end{array}$ & 0,09 & 0,10 \\
\hline$P_{D} \mathrm{X} C_{A+1}$ & 0,03 & 0,05 & 0,10 \\
\hline$P_{D} \mathrm{X} C_{A+2}$ & 0,10 & 0,08 & 0,05 \\
\hline
\end{tabular}

O melhor R-Quadrado referente ao desempenho de inovação em processo foi verificado na relação descrita no ano de 2005 comparando com a lucratividade do próprio ano $(0,11)$. De modo geral os desempenhos de inovações em processo parecem estar afetando mais a lucratividade dos próprios anos do que dos anos seguintes, com exceção do ano de 2008, sendo que esta relação pareceu mais forte em 2005. A pior relação de explicação foi verificada em 2003 comparando com a lucratividade do ano de $2005(0,02)$.

Os melhores R-Quadrados referente ao desempenho de inovação em produto foram verificados nas relações descritas do ano de 2003 comparando com a taxa de crescimento em $2005(0,10)$ e em 2008 comparando com a taxa de crescimento do próprio ano $(0,10)$ e de $2009(0,10)$. De modo geral os desempenhos de inovações em produto também parecem estar afetando mais a taxa de crescimento dos próprios anos do que a dos anos seguintes, sendo que esta relação parece apresentar uma tendência de ficar mais forte à medida que o tempo passa (2008>2005>2003). A pior relação de explicação foi verificada em 2003 comparando com a lucratividade no ano de $2004(0,03)$.

Com relação às variáveis V99 (Importância das inovações em processo para o aumento da flexibilidade da produção ou da prestação de serviços) e V102 (Importância das inovações em processo para a redução do consumo de matérias-primas), foi verificado que quanto maior a 
importância destes indicadores, menor foi a lucratividade das empresas; porém com as variáveis V98 (Importância das inovações em processo para o aumento da capacidade de produção ou de prestação de serviços) e V103 (Importância das inovações em processo para a redução do consumo de energia) ocorreu o contrário. Em outras palavras, inovações em processo que aumentaram a capacidade produtiva e/ou reduziram o consumo de energia tiveram maior efeito na lucratividade do que aquelas que aumentaram a flexibilidade da produção e/ou reduziram o consumo de matérias-primas. Esse último resultado contraria o senso comum, já que o setor de máquinas e equipamentos trabalha com produção em lotes que demanda flexibilidade do setor produtivo.

Com relação à V87 (Participação de produto novo para o mercado mundial, nas vendas líquidas internas), quanto maior a participação de produtos novos para o mercado mundial nas vendas internas, maior foi o crescimento das empresas. Porém na análise da V86, verifica-se que quanto maior a participação de produto novo ou significantemente aprimorado para o mercado nacional, mas já existente no mercado mundial nas vendas internas, menor foi o crescimento das empresas. Esta constatação pode sugerir que inovações radicais no setor de máquinas e equipamentos contribuem mais do que as inovações incrementais para o crescimento das vendas. Sobre a V94 (Importância das inovações em produto para a ampliação da gama de bens ou serviços ofertados) verifica-se que quanto maior a importância deste indicador, menor foi o crescimento da empresa. Uma possível explicação para esse achado pode ser a própria característica do setor cuja trajetória de inovação tecnológica é baseada em encomendas para atender às necessidades específicas de usuários.

\section{Setor 2 - Fabricação de produtos químicos}

\section{Tabela 6}

R-Quadrados do Setor 2 - Fabricação de produtos químicos

\begin{tabular}{|c|c|c|c|}
\hline REGRESSÕES & $\mathbf{2 0 0 3}$ & $\mathbf{2 0 0 5}$ & $\mathbf{2 0 0 8}$ \\
\hline$P_{C} \mathrm{X} L_{A}$ & 0,05 & 0,20 & 0,09 \\
\hline$P_{C} \mathrm{X} L_{A+1}$ & 0,12 & 0,37 & 0,08 \\
\hline$P_{C} \mathrm{X} L_{A+2}$ & 0,11 & 0,16 & 0,11 \\
\hline$P_{D} \mathrm{X} C_{A}$ & $\begin{array}{c}\text { Regressão não } \\
\text { realizada }\end{array}$ & 0,06 & 0,07 \\
\hline$P_{D} \mathrm{X} C_{A+1}$ & 0,24 & 0,03 & 0,07 \\
\hline$P_{D} \mathrm{X} C_{A+2}$ & 0,14 & 0,10 & 0,09 \\
\hline
\end{tabular}


Com relação a V102 (Importância das inovações em processo para a redução do consumo de matérias-primas) nos anos de 2005 e 2008, quanto maior a importância das inovações em processo para redução do consumo de matéria-prima, maior foi a lucratividade da empresa. Esta mesma relação ocorre com a V100 (Importância das inovações em processo para a redução dos custos de produção ou dos serviços prestados). Já na V103 (Importância das inovações em processo para a redução do consumo de energia) e V104 (Importância das inovações em processo para a redução do consumo de água) esta relação é inversa. Ou seja, a lucratividade das empresas foi maior quando foi dada uma maior importância às inovações em processo para redução do consumo de matéria-prima e dos custos de produção e não para a redução de energia e de água. Uma possível explicação seria os baixos custos da energia e da água no Brasil fazendo com que os benefícios financeiros de investimentos em processos para redução de desperdício de água e energia não compensem os custos dos investimentos.

Para a V85 (Participação de produto novo ou significativamente aprimorado para a empresa, mas já existente no mercado nacional, nas vendas líquidas internas), quanto maior a participação de produto novo ou significativamente aprimorado para a empresa, mas já existente no mercado nacional, nas vendas internas, maior foi o crescimento da empresa. Em contraponto a análise da V89 (Participação de produto novo ou significativamente aprimorado para a empresa, mas já existente no mercado nacional, nas exportações) nos informa que quanto maior a participação desse tipo de produto, nas exportações, menor foi o crescimento da empresa. Tal resultado pode ser explicado pelo fato desses indicadores se referirem a inovações de baixo grau de novidade mais valorizadas no mercado doméstico do que em países mais desenvolvidos para onde estejam sendo importados. Na V94 (Importância das inovações em produto para a ampliação da gama de bens ou serviços ofertados) verifica-se que quanto maior a importância deste indicador, menor foi o crescimento da empresa, conforme constatado também no Setor 1. Uma possível explicação para esse achado pode ser a própria característica do setor cuja trajetória de inovação tecnológica é marcada pelo desenvolvimento apenas de produtos tecnologicamente relacionados.

\section{Setor 3 - fabricação de equipamentos de informática, produtos eletrônicos e ópticos}

\section{Tabela 7}

R-Quadrados do Setor 3 - Fabricação de equipamentos de informática, produtos eletrônicos e ópticos

\begin{tabular}{|l|l|l|l|}
\hline REGRESSÕ்S & $\mathbf{2 0 0 3}$ & $\mathbf{2 0 0 5}$ & $\mathbf{2 0 0 8}$ \\
\hline$P_{C} \mathrm{X} L_{A}$ & 0,20 & 0,10 & 0,04 \\
\hline$P_{C} \mathrm{X} L_{A+1}$ & 0,08 & 0,09 & 0,04 \\
\hline
\end{tabular}




\begin{tabular}{|l|l|l|l|}
\hline$P_{C} \mathrm{X} L_{A+2}$ & 0,10 & 0,09 & 0,07 \\
\hline$P_{D} \mathrm{X} C_{A}$ & $\begin{array}{c}\text { Regressão } \\
\text { ealizada }\end{array}$ & 0,14 & 0,06 \\
\hline$P_{D} \mathrm{X} C_{A+1}$ & 0,05 & 0,22 & 0,05 \\
\hline$P_{D} \mathrm{X} C_{A+2}$ & 0,10 & 0,09 & 0,10 \\
\hline
\end{tabular}

Com relação à variável V100 (Importância das inovações em processo para a redução dos custos de produção ou dos serviços prestados), no ano de 2005 foi verificado que quanto maior a importância das inovações em processo para a redução dos custos de produção ou dos serviços prestados, maior foi a lucratividade. Na V103 (Importância das inovações em processo para a redução do consumo de energia) a relação com a lucratividade continua sendo positiva, já na V104 (Importância das inovações em processo para a redução do consumo de água) esta relação é inversa. Ou seja, a lucratividade das empresas foi maior quando foi dada uma maior importância às inovações em processo para redução dos custos de produção e redução do consumo de energia, ocorrendo o contrário com relação à redução do consumo de água. Conforme já explicado na análise do setor de fabricação de produtos químicos, uma possível explicação seria os baixos custos da energia e da água no Brasil que fazem com que os benefícios financeiros de investimentos em processos para redução de desperdício de água e energia não compensam os custos dos investimentos.

Na análise da V85 (Participação de produto novo ou significativamente aprimorado para a empresa, mas já existente no mercado nacional, nas vendas líquidas internas), quanto maior a participação das inovações incrementais (inovação já existente no mercado nacional) nas vendas internas, maior foi o crescimento da empresa. Em posição oposta, na análise da V94 (Importância das inovações em produto para a ampliação da gama de bens ou serviços ofertados) verifica-se que quanto maior a importância das inovações em produto para ampliação da gama de bens ou serviços ofertados, menor foi o crescimento das empresas. Esse último resultado sugere que os efeitos de diversificação da pauta de produtos ou serviços decorrentes de inovações em produtos não estão colaborando para o crescimento neste setor. No caso do setor de informática, uma possível explicação para esse achado pode ser a própria característica desse setor que, à semelhança do setor de máquinas, trabalha bastante com o sistema de encomendas, procurando monitorar e responder às necessidades específicas de usuários. Com relação aos setores de eletrônica e ótica, uma possível explicação para esse achado pode ser a própria característica do setor que, similarmente ao que ocorre no setor químico, procura desenvolver apenas produtos tecnologicamente relacionados. 


\subsection{Atendimento aos objetivos da pesquisa}

\subsubsection{Objetivo principal: a relação entre inovação e desempenho financeiro.}

Era de se esperar que os resultados encontrados nas regressões não tivessem um nível de explicação alto, tendo em vista que este trabalho não tinha a pretensão de explicar toda variação de lucratividade e crescimento apenas pelos indicadores de desempenho de inovações. De acordo com Tidd e Bessant (2009), a variância em desempenho financeiro de organizações (medida em termos de market share, lucratividade e receita) é apenas parcialmente explicada pelo desempenho de suas inovações em produto. Outros fatores também são determinantes como é o caso do tipo de mercado a ser servido (categorizado em temos de tamanho, taxa de crescimento e nível de competição) e do desempenho do processo de inovação da organização (medido em termos de lead time e produtividade).

Por outro lado, o desempenho financeiro de um determinado ano, 2006, por exemplo, pode ser influenciado por inovações em produto e processo implementadas em 2006 e vários anos anteriores (2005, 2004, 2003, 2002 etc), em função das características do processo de difusão (adoção) de inovações (Tidd \& Bessant, 2009), e não apenas pelas inovações implementadas no período 2003-2005 da PINTEC 2005 que antecedeu 2006. Ou seja, seria de se esperar que o poder de explicação (R Quadrado) do desempenho de inovações em processos e produtos desenvolvidos durante a janela de apenas três anos não fosse grande.

As conclusões acima também corroboram em parte com os resultados encontrados por Brito et al. (2009), Mauro e Brito (2011), Kemp et al. (2003) e Queiroz (2010), que também não conseguiram estabelecer uma relação positiva entre inovação e lucratividade, porém os três primeiros autores encontraram em seus resultados indícios de que pode haver uma correlação positiva entre a inovação e a taxa de crescimento das empresas. Tal fato pode ser explicado pela diferença entre tais trabalhos e a presente pesquisa, no que se refere aos indicadores que foram utilizados para medir o desempenho da inovação.

Brito et al. (2009) também utilizaram o ROA para medir a lucratividade das empresas, porém as variáveis usadas para mensurar a inovação são diferentes das utilizadas neste estudo: percentagem de gastos com P\&D; percentagem de gastos com aquisição externa de $\mathrm{P} \& \mathrm{D}$; percentagem de gastos com aquisição externa de outros conhecimentos; Pessoal ocupado com inovação - Doutores, etc.

Revista de Administração e Inovação, São Paulo, v. 12, n.3 p. 183-208, jul./set. 2015. 
Algumas variáveis utilizadas por Brito et al.(2009) são parecidas com as utilizadas por Kemp et al. (2003): percentagem de contratados com mestrado, percentagem de contratados que receberam treinamento financiado pela empresa, percentagem de tempo total gasto com inovação pelos contratados, cooperação com outras empresas, etc.

Da mesma forma, Queiroz (2010) também utilizou indicadores diferentes para medir a inovação. O autor usou os gastos com pesquisa e desenvolvimento (P\&D), classificados como "ativos de inovação" para serem relacionados com a taxa de crescimento dos lucros das empresas brasileiras, sendo que esta relação não foi comprovada.

Os resultados da presente pesquisa também são similares aos do estudo realizado por Miranda et al. (2011) onde não foi verificada nenhuma evidência sobre a contribuição do grau de inovação para a melhoria no desempenho econômico empresarial, contrariamente ao que foi encontrado nos estudos de Malachias e Meirelles (2009), Camara e Galão (2006), Benedetti (2006) e Boscolo e Sbragia (2008) que correlacionam positivamente a inovação com o desempenho financeiro das empresas.

Verifica-se, portanto, de acordo com os estudos empíricos levantados, que as pesquisas sobre a relação da inovação com o desempenho financeiro das empresas apresentam conclusões contraditórias. Em parte, as contradições derivam da utilização de construtos diferentes para as variáveis envolvidas. Como foi definido o desempenho de inovações em processo? E o desempenho das inovações em produtos? Como foram medidos a lucratividade e o crescimento?

\subsubsection{Primeiro objetivo secundário: comparação da influência do desempenho de inovações no desempenho financeiro entre os anos 2003, 2005 e 2008.}

Objetivando obter uma tendência temporal, foram comparados os valores médios dos RQuadrados de cada um dos anos (2003, 2005 e 2008) e as quantidades de variáveis explicativas com valores-P relevantes $(<10 \%)$ em cada ano. Ressalve-se que as médias de R- Quadrados foram utilizadas somente no sentido de ilustrar de uma maneira geral as comparações entre os anos e os setores (próxima seção), não podendo ser utilizadas como uma informação decisiva de resultado.

No tocante a inovações em processo, verificou-se que o ano de 2005 apresentou a melhor média de R-Quadrados (0,14 contra 0,09 de 2003 e 0,07 de 2008, conforme podem ser calculadas a partir da metade superior das Tabelas 5, 6 e 7 anteriores), mostrando que neste ano ocorreu uma maior influência do desempenho de inovações em processo na lucratividade das empresas. Da mesma forma, a maior quantidade de valores-P relevantes na relação desempenho de inovações em processo versus 
A influência da inovação em produtos e processos no desempenho de empresas brasileiras

lucratividade, ocorreu também no ano de 2005 (12 valores-P relevantes), depois em 2003 (3 valores-P relevantes) e por último em 2008 (2 valores-P relevantes).

Nas regressões de desempenho de inovações em produto versus crescimento, a partir do cálculo envolvendo os valores da metade inferior das Tabelas 5, 6 e 7 anteriores, observou-se que o ano de 2003 apresentou a melhor média de R-Quadrados $(0,11)$, mostrando que neste ano ocorreu uma maior influência do desempenho de inovações em produto no crescimento das empresas, porém esta média ficou muito próxima a média do ano de $2005(0,10)$ e não muito distante da de 2008 (0,08). Da mesma forma, a maior quantidade de valores-P relevantes na relação desempenho de inovações em produto versus crescimento, ocorreu no ano de 2003 (5 valores-P relevantes), depois em 2005 e 2008, sendo que estes dois anos totalizaram apenas 3 Valores-P relevantes cada.

\subsubsection{Segundo objetivo secundário: comparação da influência do desempenho de inovações no desempenho financeiro entre os três setores pesquisados.}

Com a finalidade de avaliar influências setoriais no que concerne à relação inovação/desempenho financeiro, foram comparados os valores médios dos R-Quadrados e as quantidades de variáveis explicativas com valores-P relevantes $(<10 \%)$ encontrados em cada um dos três setores.

A partir da comparação dos valores médios dos R-Quadrados, observou-se que a influência tanto do desempenho de inovações em processo na lucratividade como do desempenho de inovações em produto no crescimento das empresas, no Setor 2 - Fabricação de produtos químicos (médias de 0,14 e 0,10, respectivamente) foi maior que no Setor 1 (médias de 0,07 e 0,08, respectivamente) e que no Setor 3 (médias de 0,09 e 0,10, respectivamente), embora os valores encontrados em ambos os casos ainda sejam muito baixos para confirmar esta relação.

Da mesma forma, a maior quantidade de Valores-P relevantes nas duas relações estudadas (inovação em produto $X$ crescimento e inovação em processo $X$ lucratividade), ocorreu nas regressões do Setor 2 (6 e 7 Valores-P relevantes, respectivamente, contra 3 e 5, no Setor 1, e 2 e 5, no Setor 3).

Os resultados acima não indicam que houve influência setorial acentuada na relação inovação/desempenho financeiro, conforme sugerido por autores referenciados (Freeman \& Soete, 1997; Malerba, 2003; Tidd, Bessant \& Pavitt, 2005; Campos \& Ruiz, 2009). Por outro lado, os resultados parecem suportar a proposição de Mintzberg, Ahlstrand e Lampel (2005) de que ações 
gerenciais específicas a cada empresa explicariam, mais do que o setor, a variância em desempenho de empresas.

\section{CONCLUSÃO}

Esta pesquisa teve como propósito investigar a influência do desempenho de inovações em produtos e processos no desempenho financeiro de empresas brasileiras em três setores, através de dados coletados das pesquisas PINTEC (2003, 2005 e 2008) e PIA (2002 a 2010).

Diferentemente de grande parte dos estudos empíricos sobre a relação inovação/desempenho financeiro, este trabalho utilizou como medidas de desempenho da inovação, o desempenho das próprias inovações (produtos/serviços) e processos (fabris/prestação de serviços). Essas medidas são o que o IBGE denomina na PINTEC de impactos da inovação. Outros estudos utilizam o esforço inovativo, patentes e/ou atividades de P\&D como indicadores de inovação. Em concordância com outros estudos empíricos o desempenho financeiro das empresas foi verificado a partir de duas vertentes, a lucratividade, medida por meio do ROA e o crescimento, por meio da receita líquida de vendas.

Os resultados do tratamento estatístico efetuado indicaram que deve ser rejeitada a hipótese: Quanto maior o desempenho de inovações em processos, maior será a lucratividade da empresa. Entre os três setores, o Setor 2 - Fabricação de produtos químicos foi o que mais se aproximou em confirmar a hipótese. Similarmente, os resultados indicam que também deve ser rejeitada a hipótese: Quanto maior o desempenho de inovações em produtos, maior será o crescimento da empresa. Novamente, entre os três setores, o Setor 2 foi mais uma vez o que mais se aproximou em confirmar a hipótese, relativamente falando.

Também relativamente falando, quando se comparam os 3 anos (2003, 2005 e 2008) nos quais foram medidos os desempenhos de inovações em produto, percebe-se que as inovações do ano 2003 foram as que mais influenciaram o crescimento. Com relação às inovações em processo, foram as do ano 2005 as que mais influenciaram a lucratividade. Inovações relevantes em processo em 2005 parecem ter sucedido as inovações relevantes em produtos que ocorreram em 2003, convergindo em certa medida com o modelo de Abernathy e Utterback (1978).

Conforme a teoria visitada mostrou, era esperado que os resultados encontrados não tivessem um nível de significância alto, tendo em vista que há outros fatores que explicam a variância do desempenho financeiro das empresas. Constatou-se também que há discrepância entre resultados de 
estudos empíricos sobre a relação inovação versus desempenho financeiro. Esses resultados contraditórios se devem em grande parte, à extensa lista de variáveis utilizadas na mensuração do desempenho tanto da inovação quanto financeiro.

Além da rejeição das hipóteses da pesquisa, a análise das variáveis relevantes (com valor $\mathrm{P}<$ 10\%) conduzida na seção 4.1.2 sugere caminhos para o gestor em tomadas de decisão relacionadas ao processo de inovação. Entre eles podem ser citados:

- No setor de fabricação de máquinas e equipamentos, inovações em processo que aumentam a capacidade produtiva e/ou reduzam o consumo de energia terão maior efeito na lucratividade do que aquelas que aumentam a flexibilidade da produção e/ou reduzam o consumo de matérias-primas.

- Inovações radicais no setor de máquinas e equipamentos contribuem mais do que as inovações incrementais para o crescimento das vendas.

- $\quad$ O fato da trajetória de inovação tecnológica do setor de fabricação de máquinas ser marcada pela fabricação sob encomenda para atender às necessidades específicas de usuários pode explicar a contribuição negativa de inovações em produto (diversificação visando a ampliação da gama de bens ou serviços ofertados) para a lucratividade.

- Nos setores de fabricação de produtos químicos e de fabricação de equipamentos de informática, produtos eletrônicos e ópticos, a lucratividade das empresas será maior quando for dada uma maior importância às inovações em processo para redução do consumo de matéria-prima e dos custos de produção e não para a redução de energia e de água. Uma possível explicação seria os baixos custos da energia e da água no Brasil que fazem com que os benefícios financeiros de investimentos em processos para redução de desperdício de água e energia não compensam os custos dos investimentos.

- No setor de fabricação de produtos químicos, inovações de baixo grau de novidade vendidas no mercado doméstico contribuem mais para o crescimento do que quando são exportadas.

- $\quad$ O fato da trajetória de inovação tecnológica do setor de fabricação de produtos químicos ser marcada pelo desenvolvimento apenas de produtos tecnologicamente relacionado pode explicar a contribuição negativa de inovações em produto (diversificação visando a ampliação da gama de bens ou serviços ofertados) para a lucratividade.

- No setor de fabricação de equipamentos de informática, produtos eletrônicos e ópticos de os efeitos de diversificação da pauta de produtos ou serviços decorrentes de inovações em produtos não estão colaborando para o crescimento neste setor. No caso do setor de informática, uma possível explicação para esse achado pode ser a própria característica desse setor que, à semelhança do setor de 
máquinas, trabalha bastante com o sistema de encomendas, procurando monitorar e responder às necessidades específicas de usuários. Com relação aos setores de eletrônica e ótica, uma possível explicação para esse achado pode ser a própria característica do setor que, similarmente ao que ocorre no setor químico procura desenvolver apenas produtos tecnologicamente relacionados.

Além das limitações inerentes ao procedimento estatístico e do processo de coleta de dados, o estudo também sofreu limitações específicas devido ao fato das análises terem sido realizadas nas dependências do IBGE, por imposição do próprio Instituto, não sendo possível realizar mais testes posteriormente com outras variáveis ou em outros setores. Outra limitação importante é a impossibilidade de se generalizar os resultados obtidos, pois foram analisados apenas três setores. Os resultados também podem ter sido influenciados não somente por mudanças do contexto econômico entre 2002 e 2010 como também pela variabilidade das amostras em diferentes anos, tendo em vista que as bases de dados da PIA e PINTEC sofreram alterações durante o período estudado. Outra limitação importante foi a falta de dados de desempenho de inovação dos anos de 2001, 2002, 2004, 2006 e 2007, tendo em vista que a PINTEC é uma pesquisa trienal e não ocorreu nesses anos, impossibilitando uma comparação mais robusta dos dados.

\section{REFERÊNCIAS}

Abernathy, W. J., \& Utterback, J. M. (1978). Patterns of industrial innovation. Technology Review.

Andia, L. H., Garcia, R., \& Bacha, C. J. C. (2012). A Influência dos Fatores Econômicos e Jurídicos sobre o Desempenho das Empresas do Agronegócio Brasileiro. Revista de Economia e Sociologia Rural, 49(04), 875-908.

Antony, R. N., \& Govindarajan, V. (2008). Sistemas de controle gerencial (12a ed.). São Paulo: McGraw-Hill.

Arbix, G. (2010). Estratégias de inovação para o desenvolvimento. Tempo Social: revista de sociologia da USP, 22(2).

Benedetti, M. A. (2006, outubro). Inovação como Fator de Crescimento de Pequenos Negócios. Anais do Simpósio de gestão da inovação tecnológica, Gramado, RS, Brasil, 24.

Boscolo, R., \& Sbragia, R. (2008, outubro) Estratégia, Inovação e Desempenho: Uma análise da relevância da Inovação de Valor no desempenho das empresas. Anais do Simpósio de Gestão da inovação tecnológica, Brasília, DF, Brasil, 25.

Brigham, E., \& Houston, J. (1999). Fundamentos da Moderna Administração Financeira (12a ed.). Rio de Janeiro: Campus. 
Brito, E. P. Z., Brito, L. A. L., \& Morganti, F. (2009). Inovação e desempenho empresarial: Lucro ou Desenvolvimento?. Revista de administração de empresas - eletrônica, 8 (1). Recuperado de http://rae.fgv.br/rae-eletronica/vol8-num1-2009/inovacao-desempenho-empresarial-lucro-oucrescimento

Camara, M. R. G., \& Galão, F. P. (2006, outubro) Inovação e Desempenho no Arranjo Produtivo Local Embrionário do Vestuário de Londrina-PR. Anais do Simpósio de Gestão da inovação tecnológica, Gramado, RS, Brasil, 24.

Campos, B., \& Ruiz, A. U. (2009). Padrões Setoriais de Inovação na Indústria Brasileira. Revista Brasileira de Inovação, 8 (1), 167-210.

Freeman, C., \& Soete, L. (1997). The Economics of Industrial Innovation (3a ed.), Londres: Pinter.

Kemp, R.G.M., Folkeringa, M., Jong, J.P.L., \& Wubben, E.F.M. (2003). Innovation and Firm Performance, SCALES - Scientific Analysis of Entrepreneurship and SMEs. Research Report H200207.

Krauter, E. (2004, agosto). Medidas de Avaliação de Desempenho Financeiro e Criação de Valor. Anais do SEMEAD - Seminários em Administração. São Paulo, SP, Brasil, 7.

Macedo, M. A. S., Sousa, A. C., Sousa, A. C. C., \& Cípola, F. C. (2009). Análise Comparativa do Desempenho Contábil-Financeiro de Empresas Socialmente Responsáveis. Pensar Contábil, 11(43), 5-23.

Malachias, C. S., \& Meirelles, D. S. (2009). Regime tecnológico, ambiente de inovação e desempenho empresarial no setor de serviços: Um estudo exploratório das empresas de tecnologia da informação. Revista de Administração e Inovação, 6(2), 58-80.

Matarazzo, D. C. (2010). Análise financeira de balanços: abordagem básica e gerencial (7ª ed.). São Paulo: Atlas.

Mauro, M., \& Brito, L. A. L. (2011, agosto). Práticas operacionais e o desempenho financeiro das empresas do setor sucro-energético em São Paulo. Anais do Simpósio de Administração da Produção, Logística e Operações Internacionais, São Paulo, SP, Brasil, 14.

Mintzberg, H., Ahlstrand, B., Lampel, J. Strategy Safari: a guided tour through the wilds of strategic management. New York: Free Press, paperback ed., 2005.

Miranda, K. F., Gallon, A. V., \& Nogueira, L. C. B. (2011, agosto). Ativos intangíveis e grau de inovação: Fatores influentes no desempenho econômico empresarial?. Anais do Simpósio de Administração da Produção, Logística e Operações Internacionais, São Paulo, SP, Brasil, 14.

Moretti, S. P., \& Sanchez, O. P. (2011, agosto). Análise do desempenho das empresas do grande ABC no período de 2001 a 2008: Uma análise multinivel. Anais do Simpósio de Administração da Produção, Logística e Operações Internacionais, São Paulo, SP, Brasil, 14.

Organização para Cooperação Econômica e Desenvolvimento - OCDE. (2005). Manual de Oslo: Diretrizes para coleta e interpretação de dados sobre inovação [manual]. 
Oliveira, A. R., Clemente, R. G., \& Caulliraux, H. M. (2009, fevereiro). Sistemas de medição de desempenho para inovação: Revisão da literatura e problemas do campo de pesquisa. Anais do Simpósio de Administração da Produção, Logística e Operações Internacionais, São Paulo, SP, Brasil, 12.

Porter, M. Estratégia competitiva: técnicas para análise de indústrias e de concorrentes. Rio de Janeiro: Campus, 2005.

Queiroz, O. R. (2010, junho). O impacto do crescimento dos gastos em P\&D na taxa de crescimento dos lucros das empresas de acordo com modelo OJ: Um estudo no mercado de capitais Brasileiro. Anais do Congresso ANPCONT, Natal, RN, Brasil, 4.

Rezende, I. C. C, Duarte, J. M. S., Silva, P. Z. P., \& Silva, G. R. (2010). Análise da performance empresarial da PETROBRAS: um estudo sob o enfoque dos indicadores econômico-financeiros. Revista Ambiente Contábil, 2 (1), 54-69.

Rogers, E. M. (2003). Diffusion of Innovations. New York: Free Press.

Tidd, J., \& Bessant, J. (2005). Managing Innovation: integrating technological market and organizational change ( $3^{\text {th }}$ ed.). Chichester: John Willey \& Sons Ltd.

Tidd, J., Bessant, J., \& Pavitt, K. (2009) Managing Innovation: integrating technological market and organizational change ( ${ }^{\text {th }}$ ed.). Chichester: John Willey \& Sons Ltd.

Tigre, P. B. (2006). Gestão da inovação: a economia da tecnologia no Brasil. Rio de Janeiro: Elsevier.

Veloso, G. G., \& Malik, A. M. (2010). Análise do desempenho econômico-financeiro de empresas de saúde. Revista de Administração de Empresas - eletrônica, 9 (1). Recuperado de http://rae.fgv.br/rae-eletronica/vol9-num1-2010/analise-desempenho-economico-financeiroempresas-saude 


\title{
THE INFLUENCE OF PRODUCT AND PROCESS INNOVATION OVER THE PERFORMANCE OF BRAZILIAN COMPANIES
}

\begin{abstract}
The main objective of this research was to evaluate the relationship between performance of product innovations (processes) and growth (profitability) of Brazilian companies from three sectors: manufacturing of machinery and equipment; manufacturing of chemical products and manufacturing of computing equipment, electronic and optical products. We used research data of PINTEC (2003, 2005 and 2008) and PIA (2003-2010) collected at the Brazilian Institute of Geography and Statistics IBGE. We verified, based on results of multiple regression and statistical analysis, the absence of a positive relationship between the performance of process innovation (product) and profitability (growth). To some extent it was expected that innovations do not provide a high level of explanation for the variance in financial performance of companies considering that there are other factors that help explain this variance (market attractiveness, dominance of input sources, to name just two examples). It was noticed that the literature examined is also inconclusive on the relationship between innovation and financial performance. These contradictory results are mainly due to the diversity of variables used to measure both financial performance and innovation performance. Besides the rejection of the research hypotheses, the study revealed other relevant findings that can support the manager in the decision making process related to the innovation process.
\end{abstract}

Keywords: Product innovation; Process innovation; Financial performance; Profitability; Growth.

Data do recebimento do artigo: 08/08/2014

Data do aceite de publicação: 01/07/2015

Revista de Administração e Inovação, São Paulo, v. 12, n.3 p. 183-208, jul./set. 2015. 